\title{
Pacific Journal of
}

\section{Mathematics}

THE LEBESGUE DECOMPOSITION, RADON-NIKODYM DERIVATIVE, CONDITIONAL EXPECTATION, AND MARTINGALE CONVERGENCE FOR LATTICES OF SETS

RICHARD BRIAN DARST 


\title{
THE LEBESGUE DECOMPOSITION, RADON-NIKODYM DERIVATIVE, CONDITIONAL EXPECTATION, AND MARTINGALE CONVERGENCE FOR LATTICES OF SETS
}

\author{
RICHARD B. DARST
}

\begin{abstract}
In the setting of additive set functions defined on lattices of sets, a Lebesgue decomposition and a Radon-Nikodym derivative are constructed and characterized. In the appropriate case $\left(L_{2}\right)$, the Radon-Nikodym derivative is shown to be the conditional expectation. Finally, a martingale convergence theorem for Radon-Nikodym derivatives is obtained.
\end{abstract}

The origin of this paper was an interesting colloquium lecture given by H. D. Brunk at the University of California, Riverside, in December, 1968. Brunk's lecture dealt with a Radon-Nikodym derivative for $\sigma$-additive set functions defined on a $\sigma$-lattice of sets and applications of this Radon-Nikodym derivative to probability. An excellent interpretation of the role of $\sigma$-lattices in probability theory can be found in the papers of H. D. Brunk (c.f. [1], where additional references can be found). The purpose of this paper is to extend the underlying mathematical theory to encompass the case of additive set functions defined on lattices of sets.

Perhaps we should remind the reader that both the closed subsets of a metric space, $M$, and the open subsets of $M$ comprise lattices of subsets of $M$, so many familiar families of functions are instances of the setting with which this paper deals. For example, the bounded upper semi-continuous functions on the interval $I=[0,1]$ are the uniform limits of simple (see paragraph two of $\S 3$ ) functions which are measurable with respect to the lattice of closed subsets of $I$. If $M$ is a Borel subset of a separable complete metric space, then the analytic subsets of $M$ comprise an important sigma lattice of subsets of $M$.

Let $\mathfrak{U}$ be an algebra of subsets of a nonempty set $\Omega$ (i.e., $\Omega \in \mathfrak{U}$ and if each of $E$ and $F$ is an element of $\mathscr{U}$, then each of $E \cap F$ and $E^{c}=\Omega-E$ is an element of $\left.\mathfrak{i}\right)$.

Let $\mathscr{C}$ be a lattice of subsets of $\mathfrak{N}$ (i.e., $\mathscr{C}$ is a subset of $\mathfrak{V}$ such that $\mathscr{C}$ contains each of the empty set $\varphi$ and $\Omega$ and, moreover, $E, F \in \mathscr{C}$ imply $E \cup F, E \cap F \in \mathscr{C})$.

Let $\mathscr{F}=\left\{A \cap B^{c} ; A, B \in \mathscr{C}\right\}$, and denote by $\mathscr{A}$ the set of finite disjoint unions of elements of $\mathscr{F}$.

Let us examine $\mathscr{A}$ more closely. A finite intersection of elements of $\mathscr{A}$ is an element of $\mathscr{A}$. Moreover, if $E_{i}=A_{i} \cap B_{i}^{c}$ where 
$A_{i}$ and $B_{i} \in \mathscr{M}$, then $E_{i}^{c}=A_{i}^{c} \cup\left(A_{i} \cap B_{i}\right) \in \mathscr{A}$; thus,

$$
E^{c}=\left(\cup_{i} E_{i}\right)^{c}=\cap E_{i}^{c} \in \mathscr{A},
$$

and $\mathscr{A}$ is closed under complementation. Therefore, $\mathscr{A}$ is the algebra of subsets of $\Omega$ that is generated by $\mathscr{M}$.

Notice that if each of $E$ and $F$ is an element of $\mathfrak{X}$ (or $\mathscr{A}$ ), then $E \cup F \in \mathfrak{A}$ (or $\mathscr{A}$ ); and if each of $E$ and $F$ is an element of $\mathscr{F}$, then $E \cap F \in \mathscr{F}$.

Let each of $\lambda$ and $\mu$ be a nonnegative additive set function defined on $\mathscr{F}$.

It seems appropriate to consider briefly the implications of the assumption that, say, $\lambda$ be additive on $\mathscr{F}$. If each of $A$ and $B$ is an element of $\mathscr{M}$, then

$$
\lambda\left(A_{i} \cup B\right)=\lambda(A)+\lambda\left(A^{c} \cap B\right),
$$

(a) $\lambda(A \cup B)+\lambda(A \cap B)=\lambda(A)+\lambda(B)$, and

(b) $\lambda(\varnothing)=0$.

Results of B. J. Pettis [6] assert that a real valued function, $\lambda$, defined on a lattice, $\mathscr{C}$, has an additive extension to the algebra, $\mathscr{A}$, generated by $\mathscr{C}$ if, and only if, $\lambda$ satisfies (a) for all $A, B \in \mathscr{C}$ and (b). Moreover, the following elementary example illustrates the fact that the conditions

(a') $\lambda(A \cup B)=\lambda(A)+\lambda(B), A, B \in \mathscr{C l}, A \cap B=\varnothing$, and

(b) $\lambda(\varnothing)=0$

do not imply (a).

Example. $\Omega=\{1,2,3\}$,

$$
\begin{aligned}
& \mathscr{C}=\{\varnothing,\{1\},\{1,2\},\{1,3\}, \Omega\}, \\
& \lambda(\varnothing)=\lambda(\{1\})=0, \\
& \lambda(\{1,2\})=\lambda(\{1,3\})=1, \text { and } \\
& \lambda(\Omega)=3 .
\end{aligned}
$$

Recall that the norm, $\|\varphi\|$, of a bounded, real valued, additive set function, $\varphi$, defined on an algebra, say, $\mathscr{A}$ of subsets of $\Omega$ satisfies

$$
\begin{aligned}
\|\varphi\| & =\sup _{E \in \mathscr{S}}(|\varphi(E)|+|\varphi(\Omega-E)|) \\
& =\sup _{E \in \mathscr{Q}} \varphi(E)-\inf _{F \in \mathscr{O}} \varphi(F) \\
& =\sup \left\{\sum_{i=1}^{n}\left|\varphi\left(E_{i}\right)\right| ;\left\{E_{i}\right\}_{i=1}^{n} \text { a partition of } \Omega, E_{i} \in \mathscr{A}\right\} .
\end{aligned}
$$

Moreover, $\varphi \geqq 0 \Leftrightarrow \varphi(A) \leqq \varphi(B), A \subset B$. A definition of the integral of a simple function can be gleaned from (13).

The primary purposes of this paper are fourfold. In $\S 2$, we will decompose $\lambda$ into a part $s$ which is singular with respect to $\mu$ and a 
part $t$ which is absolutely continuous with respect to $\mu$. Section 3 is devoted to constructing and characterizing the Radon-Nikodym derivative $F=\left\{f_{n}\right\}$ of $\lambda$ with respect to $\mu$. In $\S 4$, it is shown that if $\lambda$ is the restriction to $\mathscr{F}$ of an element of $L_{2}(\Omega, \mathfrak{A}, \mu)$, then $F$, is the conditional expectation of $\lambda$. Finally, in $\S 5$ we shall establish an appropriate martingale convergence theorem.

2. The Lebesgue decomposition for lattices of sets. Let us begin this section by reviewing a few points concerning LebesgueRadon-Nikodym theorems.

When it is appropriate to apply a Lebesgue decomposition theorem to an object $u$ with respect to an object $v, u$ is split, uniquely, into an absolutely continuous part $u_{a}$ and a singular part $u_{s}$. The parts $u_{a}$ and $u_{s}$ exhibit antipodal behavior with respect to $v$; qualitatively, the local behavior of $u_{a}$ depends on the local behavior of $v$ while $u_{s}$ acts separately from $v$. Then one seeks a Radon-Nikodym theorem which applies to $u_{a}$ : one seeks to represent $u_{a}$ in terms of $v$. A LebesgueRadon-Nikodym theorem asserts not only that $u$ splits but also that $u_{a}$ can be represented in an appropriate fashion.

In [4] S. Johansen gives a definition and construction of a RadonNikodym derivative of a $\sigma$-additive set function with respect to a finite $\sigma$-additive measure on a $\sigma$-lattice.

Johansen's results are based on the fact that the Hahn decomposition remains valid in his setting. However, in the case of algebras of sets it is possible to have a bounded and finitely additive set function on a $\sigma$-algebra for which no Hahn decomposition exists, and it is possible to have a bounded $\sigma$-additive set function on an algebra of sets for which no Hahn decomposition exists. Nevertheless, in dealing with additive set functions on algebras of sets, approximations to Hahn decompositions ( $\varepsilon$-decompositions) exist and can be used to obtain a Lebesgue-Radon-Nikodym theorem (cf. [2]).

In this section we shall show that the $\varepsilon$-decomposition approach used in [2] carries over to lattices of sets and permits us to obtain a Lebesgue decomposition. However, in $\S 3$, by a simple example, we illustrate the fact that it is impossible to establish a general Radon-Nikodym theorem for lattices of sets. Our example shows that even in the $\sigma$-additive, $\sigma$-algebra setting of Johansen's paper the Radon-Nikodym derivative may not represent the absolutely continuous part of $\lambda$. Nevertheless, in $\S 3$ we shall refine the elementary construction of this section and obtain a Radon-Nikodym derivative for lattices of sets. In $\S 4$ we show that the Radon-Nikodym derivative of $\lambda$ represents the best $L_{2}$ approximation to $\lambda$ by $\mathscr{C} /$-measurable functions.

Definition ( $\varepsilon$-decomposition). Suppose that $\nu$ is a finitely additive 
set function on $\mathscr{F}$ which is bounded above. Let $\varepsilon>0$. Let $K \epsilon$ $\mathscr{F}$. Let $A \in \mathscr{M}$ such that $\nu(A \cap K)>\sup _{E \in \mathscr{M}} \nu(E \cap K)-\varepsilon$. Then for each $B \in \mathscr{L}, \nu\left(K \cap A \cap B^{c}\right)=\nu(K \cap A)-\nu(K \cap A \cap B)>-\varepsilon$ and $\nu\left(K \cap A^{c} \cap B\right)=\nu(K \cap[A \cup B])-\nu(K \cap A)<\varepsilon$. We shall call $K \cap A$ an $\varepsilon$-positive set for $\nu$ in $K, K \cap A^{c}$ an $\varepsilon$-negative set for $\nu$ in $K$, and $\left(K \cap A, K \cap A^{c}\right)$ an $\varepsilon$-decomposition for $\nu$ in $K$.

In order to obtain a Lebesgue decomposition of $\lambda$ with respect to $\mu$ by splitting off the singular part of $\lambda$, we introduce the following simple construction.

For each positive integer $n$, let $\varepsilon_{n}=(64)^{-(n+1)}$ and let $\left([n],[n]^{c}\right)$ be an $\varepsilon_{n}$-decomposition for $\lambda-n \mu$ in $\Omega$.

Let $s_{n}$ be the restriction of $\lambda$ to $[n]$ (i.e., $s_{n}(E)=\lambda(E \cap[n])$, $E \in \mathscr{F})$.

We shall establish two lemmas to show that $\left\{s_{n}\right\}$ is a Cauchy sequence and, hence, $\left\{s_{n}\right\}$ converges to a nonnegative bounded additive function $s=\lambda_{s}$ on $\mathscr{F}$. The restrictions $t_{n}=\lambda-s_{n}$ then converge to a nonnegative bounded additive function $t=\lambda_{a} ; s$ and $t$ will be shown to comprise that Lebesgue decomposition of $\lambda$ with respect to $\mu$.

Lemma 1. Let $m>n$, let $M$ denote $[m]$ and let $N$ denote $[n]$. Then $\lambda\left(M \cap N^{c}\right) \rightarrow 0$.

Proof. From the construction and the definitions follow

$$
(\lambda-m \mu)\left(M \cap N^{c}\right)>-\varepsilon_{m} \text { and }(\lambda-n \mu)\left(M \cap N^{c}\right)<\varepsilon_{n} .
$$

Hence

$$
m \mu\left(M \cap N^{c}\right)-\varepsilon_{m}<\lambda\left(M \cap N^{c}\right)<n \mu\left(M \cap N^{c}\right)+\varepsilon_{n},
$$

which implies

$$
\mu\left(M \cap N^{c}\right)<\left(\varepsilon_{m}+\varepsilon_{n}\right) /(m-n) \leqq \varepsilon_{m}+\varepsilon_{n},
$$

and, in turn,

$$
\lambda\left(M \cap N^{c}\right)<n\left(\varepsilon_{m}+\varepsilon_{n}\right)+\varepsilon_{n}=n \varepsilon_{m}+(n+1) \varepsilon_{n} .
$$

Next, we will use Lemma 1 and the following pertinent remarks to show that $\lambda\left(M^{c} \cap N\right) \rightarrow 0$.

Because $\lambda$ is nonnegative and additive, it follows that

$$
\lambda(G \cap H) \leqq \lambda(H)
$$

if $G$ and $H \in \mathscr{F}$.

If $\left\{K_{i}\right\}$ is a sequence of elements of $\mathscr{C}$ and $p$ is a positive integer, then 


$$
K_{p}=\left(K_{p} \cap K_{p-1}^{c}\right) \cup\left(K_{p} \cap K_{p-1} \cap K_{p-2}^{c}\right) \cup \cdots \cup\left(\left[\bigcap_{p \geqq i>1} K_{i}\right] \cap K_{1}^{c}\right) \cup T_{p},
$$

where

$$
T_{p}=\bigcap_{i \leqq p} K_{i}
$$

Moreover,

$$
\begin{aligned}
K_{p+1}^{c} \cap K_{p}=\left(K_{p+1}^{c} \cap\left[K_{p} \cap K_{p-1}^{c}\right]\right) \cup & \\
& \left(K_{p+1}^{c} \cap K_{p-1} \cap\left[K_{p} \cap K_{p-2}^{c}\right]\right) \cup \cdots \cup\left(K_{p+1}^{c} \cap T_{p}\right),
\end{aligned}
$$

and

$$
K_{p+1}^{c} \cap T_{p}=\left(K_{p+1} \cap T_{p}\right)^{c} \cap T_{p}=T_{p+1}^{c} \cap T_{p}
$$

LEMma 2. $\lambda\left(M^{c} \cap N\right) \rightarrow 0$.

Proof. Suppose, on the contrary, that there exists $\varepsilon>0$ and an increasing sequence $\left\{n_{i}\right\}$ of positive integers such that $5 n_{1} \varepsilon_{n_{1}}<\varepsilon$ and $\lambda\left(K_{2 i}^{c} \cap K_{2 i-1}\right) \geqq \varepsilon$, where $K_{j}=\left[n_{j}\right]$. Then

$$
\begin{aligned}
\lambda\left(T_{p+1}^{c} \cap T_{p}\right) & =\lambda\left(K_{p+1}^{c} \cap K_{p}\right)-\lambda\left(K_{p+1}^{c} \cap\left[K_{p} \cap K_{p-1}^{c}\right]\right)-\cdots \\
& \geqq \lambda\left(K_{p+1}^{c} \cap K_{p}\right)-\sum_{1 \leqq i<p} \lambda\left(K_{p} \cap K_{i}^{c}\right) \\
& >\lambda\left(K_{p+1}^{c} \cap K_{p}\right)-\sum_{1 \leqq 2<p}\left[n_{i} \varepsilon_{n_{p}}+\left(n_{i}+1\right) \varepsilon_{n_{i}}\right] \\
& >\lambda\left(K_{p+1}^{c} \cap K_{p}\right)-\varepsilon / 2 .
\end{aligned}
$$

Moreover, for each positive integer $p$,

$$
\begin{aligned}
\lambda\left(T_{1}\right) & =\sum_{i \leqq p} \lambda\left(T_{i} \cap T_{i+1}^{c}\right)+\lambda\left(T_{p+1}\right) \\
& \geqq \sum_{i \leqq p} \lambda\left(T_{i} \cap T_{i+1}^{c}\right) .
\end{aligned}
$$

Hence, choosing $k$ large and $2 k \leqq p+1$, the following contradiction is obtained, and Lemma 2 is thereby established.

$$
\begin{aligned}
\lambda\left(T_{1}\right) & \geqq \sum_{i \leqq k} \lambda\left(T_{2 i-1} \cap T_{2 i}^{c}\right) \\
& >\sum_{i \leqq k}\left(\lambda\left(K_{2 i-1} \cap K_{2 i}^{c}\right)-\varepsilon / 2\right) \\
& >k \varepsilon / 2 \\
& >\sup _{E \in \mathscr{J}} \lambda(E) .
\end{aligned}
$$

From $\lambda\left(M \cap N^{c}\right) \rightarrow 0, \lambda\left(M^{c} \cap N\right) \rightarrow 0$, and the monotonicity of $\lambda$, it follows that $\left\{s_{n}\right\}$ converges uniformly in $n$ on $\mathscr{F}$ to a function $s$ on $\mathscr{F}$ such that

(1) $s$ is a nonnegative additive function on $\mathscr{F}$, 
(2) if $\delta>0$, then there exists $E \in \mathscr{M}$ such that $\mu(E)<\delta$ and $s(\Omega-E)=s\left(E^{c}\right)=\sup _{F \in \mathscr{F}} s\left(E^{c} \cap F\right)<\delta$. Moreover, $t_{n}=\lambda-s_{n}$ converges uniformly to a function $t$ on $\mathscr{F}$ such that

(3) $t$ is a nonnegative additive set function on $\mathscr{F}$

(4) $\lambda=s+t$

(5) if $\varepsilon>0$, then there exists $\delta>0$ such that if $E \in \mathscr{M}$ and $\mu(E)<\delta$, then $t(E)<\varepsilon$.

Proof of (5). Choose $n$ such that $\sup _{E \in \mathscr{F}}\left|\left(t-t_{n}\right)(E)\right|<\varepsilon / 4$ and $\varepsilon_{n}<\varepsilon / 4$. Then choose $\delta=\varepsilon / 2 n$. If $E \in \mathscr{M}$ and $\mu(E)<\delta$, then

$$
\begin{aligned}
t(E)<\varepsilon / 4+t_{n}(E)= & \varepsilon / 4+\lambda\left(E \cap[n]^{c}\right)<\varepsilon / 4+n \mu\left(E \cap[n]^{c}\right) \\
& +\varepsilon_{n}<\varepsilon / 2+n \mu(E)<\varepsilon .
\end{aligned}
$$

Now that we have established the existence of a Lebesgue decomposition, it remains to establish uniqueness.

Proof of uniqueness. Suppose that $y$ and $z$ are bounded, additive functions on $\mathscr{F}$ such that

(i) $\lambda=y+z$,

(ii) if $\delta>0$, then there exists $E \in \mathscr{A}$ such that $\mu(E)<\delta$ and $\sup _{B \in \mathscr{T}}\left|y\left(E^{c} \cap B\right)\right|<\delta$, and

(iii) if $\varepsilon>0$, then there exists $\delta>0$ such that if $E \in \mathscr{C}$ and $\mu(E)<\delta$, then $|z(E)|<\varepsilon$.

Look at $s-y=z-t$. Let $\varepsilon>0$. Let $\delta$ be a positive number less than $\varepsilon$ such that if $E \in \mathscr{A}$ and $\mu(E)<2 \delta$ then $t(E)<\varepsilon$ and $|z(E)|<\varepsilon$. Let $E$ and $F \in \mathscr{C}$ such that $\mu(E)<\delta, s\left(E^{c}\right)<\delta, \mu\left(F^{\prime}\right)<$ $\delta$, and $\sup _{B \in \mathscr{F}}\left|y\left(F^{c} \cap B\right)\right|<\delta$. Let $K=E \cup F$. Then $\mu(K)<2 \delta$, $s\left(K^{c}\right)<\delta$, and

$$
\sup _{B \in \mathscr{F}}\left|y\left(K^{c} \cap B\right)\right|=\sup _{B \in \mathscr{F}} y\left(F^{c} \cap E^{c} \cap B\right) \leqq \sup _{A \in \mathscr{F}}\left|y\left(F^{c} \cap A\right)\right|<\delta .
$$

Let $A \in \mathscr{M}$. Then

$$
\begin{aligned}
|(s-y)(A)| & =\left|(s-y)\left(A \cap K^{c}\right)+(z-t)(A \cap K)\right| \\
& \leqq s\left(A \cap K^{c}\right)+\left|y\left(A \cap K^{c}\right)\right|+|z(A \cap K)|+t(A \cap K) \\
& <4 \varepsilon .
\end{aligned}
$$

Recall that if $\omega$ is an additive function on $\mathscr{F}$, then $A, B \in \mathscr{M}$ imply $\omega\left(A \cap B^{c}\right)=\omega(A)-\omega(A \cap B)$ and, hence,

$$
\sup _{C \in \varsubsetneqq}|\omega(C)| \leqq 2 \sup _{C \in \mathscr{M}}|\omega(C)| \text {. }
$$

Thus, $\sup _{C \in \mathscr{F}}|(s-y)(C)| \leqq 8 \varepsilon$ which implies that $s=y$ and $t=z$.

3. The Radon-Nikodym derivative. In this section, we shall 
construct a Radon-Nikodym derivative of $\lambda$ with respect to $\mu$. In order to describe what we shall construct, it is necessary to introduce the following notation.

A (real valued) function $f$ on $\Omega$ is said to be $\mathscr{C}$-measurable if $(f>r)=\{x ; f(x)>r\} \in \mathscr{M}$ whenever $r \in R$, the set of real numbers. If $f$ is a $\mathscr{C}$-measurable function on $\Omega$ and the range of $f$ is a finite subset of $R$, then $f$ is said to be a simple $\mathscr{C}$-measurable function.

Suppose that $\mathscr{S}$ is an algebra of subsets of $\Omega$, and that $\rho$ is a nonnegative additive set function on $\mathscr{S}$. Let $L_{p}(\Omega, \mathscr{S}, \rho), p \geqq 1$, denote the space of functions $f$ on $\Omega$ such that if $\varepsilon>0$, then there exists an $\mathscr{S}$-measurable function $g$ and an $\mathscr{S}$-measurable function $h$ such that

(i) $\int|g|^{p} d \rho<\infty$,

(ii) $|f-g| \leqq h$, and

(iii) $\int|h|^{p} d \rho<\varepsilon$.

The spaces $L_{p}(\Omega, \mathscr{S}, \rho)$ are not, in general, complete unless $\mathscr{S}$ is a $\sigma$-algebra of subsets of $\Omega$ and $\rho$ is countably additive on $\mathscr{S}$. The completions $V_{p}(\Omega, \mathscr{S}, \rho)$ of $L_{p}(\Omega, \mathscr{S}, \rho)$ are spaces of additive set functions on $\mathscr{S}$. These additive set functions can be identified with sequences $\left\{g_{n}\right\}$ of simple $\mathscr{S}$-measurable functions such that

(i) $\int\left|g_{n}\right|{ }^{p} d \mu<\infty$ and

(ii) $\int\left|g_{m}-g_{n}\right|^{p} d \mu \rightarrow 0$,

and we shall often identify the elements of the $V_{p}$-spaces that we will encounter with appropriate Cauchy sequences of simple functions. Primary sources of information about such $L_{p}$ and $V_{p}$ spaces are [3], [4], and [7].

If $\mathscr{C}$ were an algebra of subsets of $\Omega$, then it would follow from [2] that there would exist a sequence $\left\{f_{n}\right\}$ of simple $\mathscr{C}$-measurable functions such that

$$
\left\|t-\int f_{n} d \mu\right\|_{\infty} \rightarrow 0 .
$$

The following example shows that even if $\mathscr{C}$ is a $\sigma$-lattice of subsets of $\Omega$ and each of $\lambda$ and $\mu$ is $\sigma$-additive on $\mathscr{F}$, then it may not be possible to uniformly approximate $t$ by integrals of simple $\mathscr{C}$ measurable functions.

EXAMPLE. Let $\Phi$ be the union of two one element sets $a$ and $b$. Let $\mathscr{C}$ be comprised of $\varphi, a$, and $\Omega$. Then $\mathscr{C}^{c}=\{\varphi, b, \Omega\}$ and $\mathscr{F}=\{\varphi, a, b, \Omega\}$. Let $\lambda$ and $\mu$ be defined on $\mathscr{F}$ by $\lambda(\varphi)=\mu(\varphi)=0$, $\lambda(a)=2, \lambda(b)=4, \lambda(\Omega)=6, \mu(a)=\mu(b)=1, \mu(\Omega)=2$. A function $f$ 
on $\Omega$ is $\mathscr{C}$-measurable if, and only if, $f(a) \geqq f(b)$. Hence if $f$ is a $\mathscr{C}$-measurable function on $\Omega$, and $F$ is defined on $\mathscr{F}$ by

$$
F(E)=\int_{E} f d \mu,
$$

then $F(a) \geqq F(b)$. Thus, $\lambda$ cannot be uniformly approximated by integrating $\mathscr{C}$-measurable functions with respect to $\mu$. But, $\lambda$ is absolutely continuous with respect to $\mu$.

The Radon-Nikodym derivative of $\lambda$ with respect to $\mu$ will be a $L_{1}$-Cauchy sequence $\left\{f_{n}\right\}$ of simple $\mathscr{C}$-measurable functions with properties that will be discussed. The Radon-Nikodym derivative $F=$ $\left\{f_{n}\right\}$ of $\lambda$ is then absolutely continuous with respect to $\mu$, and it is reasonable to ask whether $F$ is the Radon-Nikodym derivative of the absolutely continuous part $t$ of $\lambda$. The answer to this latter question is yes (see (14) and the definition of $t$ ).

The construction of the sequence $\left\{f_{n}\right\}$ is fairly straightforward; but, a direct proof that it is a Cauchy sequence appears to involve a dreadful computation which we shall avoid by complicated but conceptually reasonable means.

To simplify the notation, we often denote $(x / y) \mu$ by $x / y \mu$ when each of $x$ and $y$ is a real number. For example, $n \mu=n 2^{n} / 2^{n} \mu$.

CONSTRUCTION. Let $n$ be a positive integer. We wish to refine the construction that we used to establish a Lebesgue decomposition. Recall that $\varepsilon_{n}=(64)^{-(n+1)}$ and that $N_{n 2^{n}}=N=[n]$ is an $\varepsilon_{n}$-positive set for $(\lambda-n \mu)=\left(\lambda-n 2^{n} / 2^{n} \mu\right)$ in $\Omega$. For $n 2^{n}>i \geqq 1$, let $N_{i} \in \mathscr{M}$ such that $N_{i} \cap\left(\bigcap_{n 2^{n} \geqq j>i} N_{j}^{c}\right)$ is an $\varepsilon_{n}$-positive set for $\left(\lambda-i / 2^{n} \mu\right)$ in $\bigcap_{n 2^{n} \geqq j>i} N_{j}^{c}$. Notice that we can assume that

$$
\varphi=N_{n 2^{n}+1} \subset N_{n 2^{n}} \subset \cdots \subset N_{i} \subset \cdots \subset N_{1} \subset N_{0}=\Omega ;
$$

let us call such a sequence an $\varepsilon_{n}$-decomposition sequence for $n$.

It will greatly simplify the typography to introduce the following notation.

$$
\text { Let } L_{i}=N_{i+1}^{c} \cap N_{i} \text {. }
$$

The following are immediate consequences of the construction.

$$
\begin{array}{cc}
\left(\lambda-i / 2^{n} \mu\right)\left(L_{i} \cap B^{c}\right)>-\varepsilon_{n}, & B \in \mathscr{M}, \\
-\varepsilon_{n}+i / 2^{n} \mu\left(L_{i} \cap B^{c}\right)<\lambda\left(L_{i} \cap B^{c}\right), & B \in \mathscr{M}, \\
\left(\lambda-i / 2^{n} \mu\right)\left(N_{i}^{c} \cap A\right)<\varepsilon_{n}, & A \in \mathscr{M}, \\
\lambda\left(N_{i}^{c} \cap A\right)<i / 2^{n} \mu\left(N_{i}^{c} \cap A\right)+\varepsilon_{n}, & A \in \mathscr{H} .
\end{array}
$$


The following consequence of (9) will be applied several times in the paper; since $\left(\lambda-i / 2^{n} \mu\right)\left(N_{i+1}^{c} \cap\left[N_{i} \cap A\right]\right)=\left(\lambda-i / 2^{n} \mu\right)\left(L_{i} \cap A\right)$,

$$
\left(\lambda-i / 2^{n} \mu\right)\left(L_{i} \cap A\right)<2^{-n} \mu\left(L_{i} \cap A\right)+\varepsilon_{n} .
$$

Let $f_{n}$ be the simple $\mathscr{L}$-measurable function defined by

$$
f_{n}=2^{-n} \sum_{1 \leqq i \leqq a_{n}} \chi_{N_{i}}=\sum_{i \leqq a_{n}} i / 2^{n} \chi_{L_{i}}, \text { where } a_{n}=n 2^{n} .
$$

Let $F_{n}$ be the nonnegative additive function defined by

$$
\begin{aligned}
F_{n}(E) & =\int_{E} f_{n} d \mu \\
& =\sum_{1 \leqq i \leqq a_{n}} i / 2^{n} \mu\left(L_{i} \cap E\right) \\
& =2^{-n} \sum_{1 \leqq i \leqq a_{n}} \mu\left(N_{i} \cap E\right) .
\end{aligned}
$$

Recall that $(\lambda-n \mu)\left(N \cap A^{c}\right)>-\varepsilon_{n}, A \in \mathscr{M}$, and that

$$
(\lambda-m \mu)\left(M \cap A^{c}\right)>-\varepsilon_{m},
$$

Hence $n \mu\left(N \cap M^{c}\right)<\lambda\left(N \cap M^{c}\right)+\varepsilon_{n}$. Moreover, $m \mu(M)<\lambda(M)+\varepsilon_{m}$ which implies that $n \mu(M)<n / m\left(\lambda(M)+\varepsilon_{m}\right)$. Thus,

$$
\begin{aligned}
n \mu(N) & =n\left(\mu\left(N \cap M^{c}\right)+\mu(N \cap M)\right) \\
& <\lambda\left(N \cap M^{c}\right)+\varepsilon_{n}+n / m(\lambda(\Omega)+1) .
\end{aligned}
$$

Hence, choosing $m$ to be large and applying Lemma 2, we obtain

$$
n \mu(N) \rightarrow 0 \text {. }
$$

Recall that $t_{n}(E)=\lambda\left(E \cap N^{c}\right)=\sum_{0 \leqq i<a_{n}} \lambda\left(L_{i} \cap E\right)$; hence

$$
t_{n}(E)-F_{n}(E)=\sum_{0 \leqq i<a_{n}}\left(\lambda-i / 2^{n} \mu\right)\left(L_{i} \cap E\right)-n \mu(N \cap E) .
$$

Then (7) implies

$$
t_{n}\left(B^{c}\right)-F_{n}\left(B^{c}\right)>-a_{n} \varepsilon_{n}-n \mu(N \cap E), \quad B \in \mathscr{C} .
$$

Moreover, applying (11) to (15) yields

$$
t_{n}(A)-F_{n}(A)<a_{n} \varepsilon_{n}+2^{-n} \mu\left(N^{c} \cap A\right), \quad A \in \mathscr{M} .
$$

We wish to show that $F_{n}$ converges uniformly to a nonnegative additive function $F$ on $\mathscr{F}$. We know from (14), (16), and (17) that $F_{n}$ converges to $t$ uniformly on $\mathscr{M} \cap \mathscr{M}^{c}$. Hence if $\mathscr{C}$ were an algebra of subsets of $\Omega$, then it would follow that $F_{n} \rightarrow t$ on $\mathscr{C}=$ $\mathscr{F}$. In general, we know that $F_{n}$ need not converge to $t$. We shall establish the uniform convergence of $F_{n}$ on $\mathscr{F}$ by showing that $F_{n}$ is almost increasing on $\mathscr{F}$. (What we mean by the term "almost 
increasing" will become clear in due course.)

Let $m>n$, and let $\varphi=M_{a_{m}+1} \subset M=M_{a_{m}} \subset \cdots \subset M_{1} \subset M_{0}=\Omega$ be an $\varepsilon_{m}$-decomposition sequence for $m$.

Since $N_{i}=\bigcup_{i \leqq j \leqq a_{n}} L_{j}$, it follows from (8) that for $A \in \mathscr{M}$,

$$
\begin{aligned}
-a_{n} \varepsilon_{n}+i / 2^{n} \mu\left(N_{i} \cap A^{c}\right) & =-a_{n} \varepsilon_{n}+\sum_{i \leqq j \leqq a_{n}} i / 2^{n} \mu\left(L_{j} \cap A^{c}\right) \\
& \leqq-a_{n} \varepsilon_{n}+\sum_{i \leqq j \leqq a_{n}} j / 2^{n} \mu\left(L_{j} \cap A^{c}\right) \\
& <\sum_{i \leqq j \leqq a_{n}} \lambda\left(L_{j} \cap A^{c}\right) .
\end{aligned}
$$

Hence,

$$
i / 2^{n} \mu\left(N_{i} \cap A^{c}\right)<a_{n} \varepsilon_{n}+\lambda\left(N_{i} \cap A^{c}\right),
$$

If $A^{c}=M_{j}^{c}$, then (10) implies that $\lambda\left(N_{i} \cap M_{j}^{c}\right)<\varepsilon_{m}+j / 2^{m} \mu\left(N_{i} \cap M_{j}^{c}\right)$. Thus,

$$
\left(i / 2^{n}-j / 2^{m}\right) \mu\left(N_{i} \cap M_{j}^{c}\right)<a_{n} \varepsilon_{n}+\varepsilon_{m} .
$$

Let $p=2^{m-n}$, and let $K_{j}=M_{j p}, j=1, \cdots, m 2^{n}$. Then (see (13))

$$
\begin{aligned}
F_{m}(E)= & 2^{-m} \sum_{i \leqq a_{m}} \mu\left(M_{i} \cap E\right)=2^{-m}\left[\left(\mu\left(M_{1} \cap E\right)+\cdots+\mu\left(M_{p} \cap E\right)\right)\right. \\
& \left.+\left(\mu\left(M_{p+1} \cap E\right)+\cdots+\mu\left(M_{2 p} \cap E\right)\right)+\cdots\right] \\
\geqq & 2^{-n}\left[\mu\left(K_{1} \cap E\right)+\mu\left(K_{2} \cap E\right)+\cdots+\mu\left(K_{m 2^{n}} \cap E\right)\right]
\end{aligned}
$$

(recall that $\left.K_{1} \supset K_{2} \supset \cdots\right)$.

In (19), let $j=(i-1) p$. Then $\left.\left(i / 2^{n}-j / 2^{m}\right)\right)=2^{-n}$, and (19) becomes

$$
\mu\left(N_{i} \cap K_{i-1}^{c}\right)<2^{n}\left(a_{n} \varepsilon_{n}+\varepsilon_{m}\right) .
$$

From (13) it follows that

$$
\begin{aligned}
F_{n}(E) & =2^{-n} \sum_{i \leqq a_{n}} \mu\left(N_{i} \cap E\right) \\
& =2^{-n} \sum_{i \leqq a_{n}}\left[\mu\left(N_{i} \cap K_{i-1}^{c} \cap E\right)+\mu\left(N_{i} \cap K_{i-1} \cap E\right)\right] \\
& \leqq 2^{-n} \sum_{i \leqq a_{n}} \mu\left(N_{i} \cap K_{i-1}^{c} \cap E\right)+2^{-n} \sum_{i \leqq a_{n}} \mu\left(K_{i-1} \cap E\right) .
\end{aligned}
$$

Hence using (20) and the paragraph between (19) and (20), we obtain

$$
\begin{aligned}
F_{n}(E) & <\delta_{n}+F_{m}(E), \text { where } \\
\delta_{n} & =2^{-n} a_{n} 2^{n}\left(a_{n} \varepsilon_{n}+\varepsilon_{m}\right)=a_{n}\left(a_{n} \varepsilon+\varepsilon_{m}\right) .
\end{aligned}
$$

Moreover, an inspection of the argument which produced (21) shows that (21) is valid if $\left\{E_{i}\right\}$ is a finite collection of pairwise disjoint elements of $\mathscr{F}, E=\bigcup_{i} E_{i}$, and $F_{n}(E)$ is defined to be $\sum_{i} F_{n}\left(E_{i}\right)$.

Inequality (21) says that $F_{n}$ is almost increasing. Since the $F_{n}$ 's 
are nonnegative and $\lim \sup _{n} F_{n}(E) \leqq \lim _{n} F_{n}(\Omega)=t(\Omega)$, the $F_{n}$ 's are uniformly bounded. Hence $F(E)=\lim _{n} F_{n}(E)$ exists for each finite union $E$ of pairwise disjoint elements of $\mathscr{F}$.

We have extended the $F_{n}^{\prime}$ 's to the set $\mathscr{A}$ of finite disjoint unions of elements of $\mathscr{F}$. Recall that $\mathscr{A}$ is the algebra of subjects of $\Omega$ which is generated by $\mathscr{C}$ or $\mathscr{F}$, and a nonnegative additive function on $\mathscr{F}$ has a unique additive extension to $\mathscr{A}$. Moreover, we have shown that the extensions converge (almost increasingly) pointwise on $\mathscr{A}$ to a nonnegative additive function $F$.

Because the $F_{n}^{\prime}$ 's are almost increasing to $F$ on $\mathscr{A}$ and $\mathscr{A}$ is an algebra of subsets of $\Omega$, it is easy to see that the $F_{n}^{\prime}$ 's coverge uniformly to $F$ on $\mathscr{A}$. But, $\mathscr{F} \subset \mathscr{A}$, and the sequence $\left\{F_{n}\right\}$ converges to $F$ in $L_{1}$-norm.

In summary, the sequence $\left\{f_{n}\right\}$ is a Cauchy sequence in $L_{1}(\Omega, \mathscr{A}, \mu)$ and the integrals $F_{n}$ of $f_{n}$ converge to $F$ uniformly.

By the Radon-Nikodym derivative of $\lambda$ with respect to $\mu$, we shall mean the object $\left\{f_{n}\right\}=F$.

We shall conclude this section with a characterization of the Radon-Nikodym $\left\{f_{n}\right\}$ that is analogous to that given by S. Johansen ([4, Th. 4]) in the case where $\mathscr{C}$ is a $\sigma$-lattice in $\mathfrak{N}$ and each of $\lambda$ and $\mu$ is countably additive on $\mathscr{F}$. From (10):

$$
\lambda\left(N_{i}^{c} \cap B\right)<i / 2^{n} \mu\left(N_{i}^{c} \cap B\right)+\varepsilon_{n}, \quad B \in \mathscr{M},
$$

and (18):

$$
\lambda\left(A^{c} \cap N_{i}\right)>i / 2^{n} \mu\left(A^{c} \cap N_{i}\right)-a_{n} \varepsilon_{n}, \quad A \in \mathscr{L},
$$

we obtain the following proposition which will be shown to characterize the Radon-Nikodym derivative.

Let $M>0$ and $\varepsilon>0$. Then there exists a positive integer $k$ such that if $n>k, a, b \in R, b<M$, and $A, B \in \mathscr{L}$, then

(i) $\quad \lambda\left(\left[f_{n} \leqq b\right] \cap A\right)<b \mu\left(\left[f_{n} \leqq b\right] \cap A\right)+\varepsilon$, and

(ii) $\lambda\left(B^{c} \cap\left[f_{n}>a\right]\right)>a \mu\left(B^{c} \cap\left[f_{n}>a\right]\right)-\varepsilon$.

THEOREM (characterization of the Radon-Nikodym derivative). Suppose that $\left\{g_{n}\right\}$ is a sequence of simple $\mathscr{C l}$-measurable functions such that $\left\{g_{n}\right\}$ is an $L_{1}$-Cauchy sequence (i.e., $\left.\int_{\Omega}\left|g_{m}-g_{n}\right| d \mu \rightarrow 0\right)$. If $\left\{g_{n}\right\}$ can play the role of $\left\{f_{n}\right\}$ in (22), then $\left\{g_{n}\right\} \equiv\left\{f_{n}\right\}$.

Proof. Suppose that $\left\{g_{n}\right\}$ can play the role of $\left\{f_{n}\right\}$ in (22). Let $\delta>0$. Let $\varepsilon$ be a positive number less than one; $\varepsilon$ will be specified later. Notice that for sufficiently large $n$, (22-(ii)) implies the inequality $\mu\left(\left[f_{n}>a\right]\right)<(\varepsilon+\lambda(\Omega)) / a$ for $f_{n}$ and the analogous inequality 
for $g_{n}$. Suppose that $a=(i+1) \delta$ and $b=i \delta$. Then for sufficiently large $n, \quad a \mu\left(\left[f_{n} \leqq b\right] \cap\left[g_{n}>a\right]\right)-\varepsilon<b \mu\left(\left[f_{n} \leqq b\right] \cap\left[g_{n}>a\right]\right)+\varepsilon$ or $\mu\left(\left[f_{n} \leqq b\right] \cap\left[g_{n}>a\right]\right)<2 \varepsilon / \delta$; symmetrically, $\mu\left(\left[g_{n} \leqq b\right] \cap\left[f_{n}>a\right]\right)<2 \varepsilon / \delta$. Choose a positive integer $m$ such that $(1+\lambda(\Omega)) / m \delta<\delta / 2$. Then, using consecutive terms of the sequence $0<\delta<2 \delta<\cdots<m \delta$ for $b$ and $a$, we obtain $\mu\left(\left[\left|f_{n}-g_{n}\right|>2 \delta\right)<(1+\lambda(\Omega)) / m \delta+2 m(2 \varepsilon / \delta)\right.$ which can be made $<\delta$ by choosing $\varepsilon$ to be sufficiently small. Hence the sequence $\left\{f_{n}-g_{n}\right\}$ converges to zero in $\mu$-measure. However, the sequence $\left\{f_{n}-g_{n}\right\}$ is an element of $V_{1}(\Omega, \mathscr{A}, \mu)$ and, hence, the integrals

$$
\int\left(f_{n}-g_{n}\right) d \mu
$$

define a weakly convergent sequence of bounded and finitely additive set functions on $\mathscr{A}$. It then follows from the proof of Theorem 2.1 in [2] that $\int_{\Omega}\left|f_{n}-g_{n}\right| d \mu \rightarrow 0$ and, hence, $\left\{f_{n}\right\} \equiv\left\{g_{n}\right\}$.

4. Conditional expectation. Suppose that $\mu$ is a nonnegative additive set function on $\mathfrak{Y}$ and that we have been looking at its restrictions to $\mathscr{C}, \mathscr{F}$, and $\mathscr{A}$.

We have already remarked that the completions $V_{p}$ of $L_{p}=$ $L_{p}(\Omega, \mathfrak{N}, \mu)$ are spaces of additive set functions on $\mathfrak{X}$ and that the Radon-Nikodym derivative $F=\left\{f_{n}\right\}$ of $\lambda$ with respect to $\mu$ is an element of $V_{1}(\Omega, \mathscr{A}, \mu)$ which extends to $V_{1}$.

Notice that since $\mu(\Omega)<\infty, V_{2} \subset V_{1}$.

Suppose that $\lambda$ is the restriction to $\mathscr{F}$ of an element $H=\left\{h_{m}\right\}$ of $V_{2}$ (i.e., $\left.H(E)=\lim _{n} \int_{E} h_{n} d \mu\right)$. Then the following theorem provides a rather satisfying extension of results of $H$. D. Brunk and others (cf. [1], [4], the references in [1] and [4], ...). Among other things, our theorem characterizes $F$ as the best $V_{2}$ approximation to $H$ that can be obtained via a $L$-Cauchy sequence of simple $\mathscr{C}$ measurable functions.

THEOREM.

(i) $F \in V_{2}$,

(ii) $\int H K d \mu \leqq \int F K d \mu, K=\left\{K_{m}\right\} \mathscr{L}$-measurable, $K \in V_{2}$,

(iii) $\int H K d \mu \geqq \int F K d \mu, K=\left\{k_{m}\right\} \mathscr{C}^{c}$-measurable, $K \in V_{2}$,

(iv) $\int H F d \mu=\int F^{2} d \mu$,

(v) $\int(H-F)^{2} d \mu \leqq \int(H-K)^{2} d \mu, K=\left\{k_{m}\right\} \mathscr{C l}$-measurable, $K \in$ 
Moreover, the conditions of the theorem characterize $F$ among the $L_{2}$-Cauchy sequences of simple $\mathscr{C}$-measurable functions.

Proof of (i). In order to establish (i), it suffices to show that

$$
\int_{\Omega}\left|f_{m}-f_{n}\right|^{2} d \mu \rightarrow 0
$$

because then there exists $G \in V_{2}(\Omega, \mathscr{A}, \mu)$ such that $G=\left\{f_{n}\right\}$ and

$$
\begin{aligned}
\|F-G\|_{1} \leqq & \left\|F-F_{n}\right\|_{1}+\left\|F_{n}-G\right\|_{1} \leqq \| F \\
& -F_{n}\left\|_{1}+(\mu(\Omega))^{1 / 2}\right\| F_{n}-G \|_{2} .
\end{aligned}
$$

We can assume, without loss of generality, that $\mu(\Omega)=1$. We shall first show that $\left\{f_{n}\right\}$ is bounded in $L_{2}$. To this end, let $\rho \in V_{1}$ be defined by

$$
\rho(E)=\lim _{n} \int_{E} h_{n}^{2} d \mu,
$$

and let $\left\{u_{n}\right\}$ be the Radon-Nikodym derivative of $\rho$ with respect to $\mu$ as constructed in $\S 3$.

For the sequence $\left\{f_{n}\right\},(22)$ can be refined to read

$$
\begin{array}{ll}
\text { (i ) } & \lambda\left(\left[f_{n} \leqq b\right] \cap A\right)<\left(b+2^{-n}\right) \mu\left(\left[f_{n} \leqq b\right] \cap A\right)+\varepsilon_{n}, \quad A \in \mathscr{C}, \\
\text { (ii ) } & \lambda\left(\left[f_{n}>a\right] \cap B^{c}\right)>\left(a-2^{-n}\right) \mu\left(\left[f_{n}>a\right] \cap B^{c}\right)-a_{n} \varepsilon_{n}, \\
& B \in \mathscr{C} .
\end{array}
$$

Moreover, the inequalities in (23) also hold for $\left\{u_{n}\right\}$ with respect to $\rho$. These versions of (23) will be utilized in our proof that $\left\{f_{n}\right\}$ is bounded in $L_{2}$.

Let $a^{2}>b$, let $A=\left(f_{n}^{2}>a^{2}\right)$, and let $B^{c}=\left(u_{n} \leqq b\right)$. From (23) and Hölder's inequality we obtain for $C=A \cap B^{c}=\left(f_{n}^{2}>a^{2}>b \geqq u_{n}\right)$ the following chain:

$$
\begin{aligned}
{\left[\left(\alpha-2^{-n}\right) \mu(C)-a_{n} \varepsilon_{n}\right]^{2} } & <[\lambda(C)]^{2} \approx\left(\int_{C} h_{m} d \mu\right)^{2} \\
& \leqq\left(\int_{C} h_{m}^{2} d \mu\right) \mu(C) \approx \rho(C) \mu(C) \\
& <\left[\left(b+2^{-n}\right) \mu(C)+\varepsilon_{n}\right] \mu(C) .
\end{aligned}
$$

Rearranging the first and last terms of the chain leads to

$$
\left[\left(a-2^{-n}\right)^{2}-\left(b+2^{-n}\right)\right][\mu(C)]^{2}<\left[2 a_{n} \varepsilon_{n}\left(a-2^{-n}\right)+\varepsilon_{n}\right] \mu(C)+\left(a_{n} \varepsilon_{n}\right)^{2} .
$$

But, $A=\varphi$ if $a^{2} \geqq n^{2}$; and if $a^{2}<n^{2}$, then

$$
a^{2}-2 a 2^{-n}+4^{-n}-b-2^{-n}>a^{2}-b-(2 n+1) 2^{-n}
$$

and $\left(a^{2}-b\right)[\mu(C)]^{2}<\xi_{n}$ where 


$$
\xi_{n}<\left(2 a_{n} \varepsilon_{n} n+\varepsilon_{n}\right) \mu(\Omega)+\left(a_{n} \varepsilon_{n}\right)^{2}+(2 n+1) 2^{-n}[\mu(\Omega)]^{2} .
$$

Then, since $\left(f_{n}^{2}>u_{n}+2\right) \subset \bigcup_{0 \leqq k<n^{2}}\left(f_{n}^{2}>k+1 \geqq k \geqq u_{n}\right)$,

$$
\mu\left(\left[f_{n}^{2}>u_{n}+2\right]\right)<n^{2}\left(\xi_{n}\right)^{1 / 2}
$$

and, hence,

$$
\int f_{n}^{2} d \mu \leqq \int u_{n} d \mu+2+n^{4}\left(\xi_{n}\right)^{1 / 2} \leqq P,
$$

where $P$ is independent of $n$. Therefore, $\left\{f_{n}\right\}$ is bounded in $L_{2}$.

Perhaps we should digress briefly and comment on the last term, $(2 n+1) 2^{-n}[\mu(\Omega)]^{2}$, of the inequality that determines $\xi_{n}$. Firstly, $2 n+1$ comes from $\left\|f_{n}\right\|_{\infty}, 2^{-n}$ comes from the mesh of $f_{n}$, and $\mu(\Omega)$ was taken to be one so this component appears only for emphasis; and secondly, the term $n^{4}\left(\xi_{n}\right)^{1 / 2}$ appears in bounding the $f_{n}^{\prime}$ 's in $L_{2}$. This is the only argument in which the ratio of $\left\|f_{n}\right\|_{\infty}$ to the mesh of $f_{n}$ has to be controlled: all the other arguments can be pushed through by adjusting $\varepsilon_{n}$.

We know that $\left\{f_{n}\right\}$ converges in $L_{1}$, and we have just shown that $\left\{f_{n}\right\}$ is bounded in $L_{2}$. Hence $\left\{f_{n}\right\}$ converges weakly in $V_{2}(\Omega, \mathscr{A}, \mu)$ : Suppose that $G \in V_{2}(\Omega, \mathscr{A}, \mu)$ and $\varepsilon>0$. Then there exists a simple $\mathscr{A}$-measurable function $k=\sum_{j \leqq v} c_{j} \chi_{E_{j}}$ such that if $K=\int k$, then $\| G-$ $K \|_{2}<\varepsilon$ and

$$
\begin{aligned}
\left|\left(G, F_{m}-F_{n}\right)\right| \leqq & \left|\left(G-K, F_{m}-F_{n}\right)\right|+\left|\left(K, F_{m}-F_{n}\right)\right| \\
& \leqq \\
& <2 \varepsilon P^{1 / 2}+\|\|_{2} \cdot\left(2 P^{1 / 2}\right)+\left|\sum_{j \leqq v} c_{j} \int_{E_{j}}\left(f_{m}-f_{m}\right) d \mu\right|
\end{aligned}
$$

Denote the weak limit of $\left\{f_{n}\right\}$ in $V_{2}(\Omega, \mathscr{A}, \mu)$ by $G$.

We know that $\|G\|_{2} \leqq \liminf _{n}\left\|f_{n}\right\|_{2}$. Suppose for the moment that $\left\|f_{n}\right\|_{2} \rightarrow\|G\|_{2}$ (i.e., $\|G\|_{2} \geqq \lim \sup _{n}\left\|f_{n}\right\|_{2}$ ). Then

$$
\left\|G-F_{n}\right\|_{2}^{2}=\left(G-F_{n}, G-F_{n}\right)=(G, G)-2\left(G, F_{n}\right)+\left(F_{n}, F_{n}\right) \rightarrow 0
$$

(i.e., $\left\{f_{n}\right\}$ converges to $G$ in $V_{2}(\Omega, \mathscr{A}, \mu)$ ) and, as we remarked before, $G=F$. Hence in order to complete a proof of (i), it suffices to show below that $\|G\|_{2} \geqq \lim \sup _{n}\left\|f_{n}\right\|_{2}$.

Because $\left\{f_{n}\right\}$ is almost increasing, using (20), we have

$$
\begin{aligned}
\mu\left(\left[f_{n}>f_{m}+2 \cdot 2^{-n}\right]\right) & \leqq \sum_{0 \leqq i<a_{n}} \mu\left(\left[f_{n}>(i+1) / 2^{n}>i / 2^{n} \geqq f_{m}\right]\right) \\
& =\sum \mu\left(N_{i+2} \cap K_{i+1}^{c}\right) \\
& <a_{n} 2^{n}\left(a_{n} \varepsilon_{n}+\varepsilon_{m}\right)=\eta_{n} .
\end{aligned}
$$

Hence, 


$$
\begin{aligned}
\int_{E} f_{n}^{2} d \mu & <\int_{E}\left(f_{m}+2^{-(n-1)}\right)^{2} d \mu+n^{2} \eta_{n} \\
& <\int_{E} f_{m}^{2} d \mu+2^{-n} \int_{E} f_{m} d \mu+4^{-(n-1)} \mu(E)+n^{2} \eta_{n},
\end{aligned}
$$

and $\left\{f_{n}^{2}\right\}$ is almost increasing. Thus, $\left\|F_{n}\right\|_{2}$ is almost increasing. Moreover, it follows from S. Leader's work ([5]) that

$$
\begin{aligned}
\left(G, F_{n}\right) & =\sum_{i \leqq a_{n}} \frac{G\left(L_{i}\right) F_{n}\left(L_{i}\right)}{\mu\left(L_{i}\right)}=\sum_{i \leqq a_{n}} i / 2^{n} G\left(L_{i}\right) \\
& =\lim _{m} \sum_{i \leqq a_{n}} i / 2^{n} F_{m}\left(L_{i}\right)\left(F_{m} \stackrel{\omega}{\rightarrow} G\right) \\
& \geqq \lim _{m} \sum_{i \leqq a_{n}} i / 2^{n}\left(F_{n}\left(L_{i}\right)-\delta_{n}\right)(\text { see }(21)) \\
& =\sum_{i \leqq a_{n}} i / 2^{n} \int_{L_{i}} f_{n} d \mu-a_{n}\left(a_{n}+1\right) 2^{n+1} \delta_{n} \\
& >\int_{\Omega} f_{n}^{2} d \mu-n^{2} 2^{n} \delta_{n} .
\end{aligned}
$$

Hence, $\left\|F_{n}\right\|_{2}^{2}-n^{2} 2^{n} \delta_{n} \leqq\left\|F_{n}\right\|_{2}\|G\|_{2}$ and, finally, $\lim \left\|F_{n}\right\|_{2}=\|G\|_{2}$.

Proof of (ii). Firstly, notice that

$$
\int H K d \mu=\lim _{m} \int H k_{m} d \mu=\lim _{m} \int k_{m} d \lambda
$$

and that

$$
\int F K d \mu=\lim _{m} \int F k_{m} d \mu=\lim _{m}\left(\lim _{n} \int k_{m} f_{n} d \mu\right) .
$$

But, fixing $m, k_{m}=\sum_{j \unlhd_{m}} b_{j} \chi_{B_{j}}, b_{j}>0, B_{j} \in \mathscr{l l}$. Hence

$$
\int k_{m} d \lambda=\sum_{j} b_{j} \lambda\left(B_{j}\right)
$$

and

$$
\int k_{m} f_{n} d \mu=\sum_{j} b_{j} \int_{B_{j}} f_{n} d \mu=\sum_{j} b_{j} \sum_{i \leqq a_{n}} i / 2^{n} \mu\left(L_{i} \cap B_{j}\right) .
$$

Thus,

$$
\begin{aligned}
& \int k_{m} d \lambda-\int k_{m} f_{n} d \mu \\
= & \sum_{j} b_{j} \sum_{i \leqq a_{n}}\left(\lambda-i / 2^{n} \mu\right)\left(L_{i} \cap B_{j}\right) \\
\leqq & \sum_{j} b_{j} \sum_{i \leqq a_{n}}\left[2^{-n} \mu\left(L_{i} \cap B_{j}\right)+\varepsilon_{n}\right](\text { see }(11)) \\
= & \sum_{j} b_{j}\left[2^{-n} \mu\left(B_{j}\right)+a_{n} \varepsilon_{n}\right] \rightarrow 0 \text { as } n \rightarrow \infty,
\end{aligned}
$$

which implies that 


$$
\int k_{m} d \lambda \leqq \lim _{n} \int k_{m} f_{n} d \mu
$$

Hence (ii) is established.

Because the proof of (iii) is analogous to that of (ii) we will omit the details.

Proof of (iv). It follows from (ii) that

$$
\int H F d \mu \leqq \int F^{2} d \mu
$$

so it will suffice to show that

$$
\int H F d \mu=\lim _{n} \int f_{n} d \lambda \geqq \lim _{n} \int f_{n}^{2} d \mu=\int F^{2} d \mu .
$$

But,

$$
\int f_{n} d \lambda=\sum_{i \geqq a_{n}} i / 2^{n} \lambda\left(L_{i}\right)
$$

and

$$
\int f_{n}^{2} d \mu=\sum_{i \leqq a_{n}}\left(i / 2^{n}\right)\left(i / 2^{n}\right) \mu\left(L_{i}\right)
$$

Hence

$$
\begin{aligned}
\int f_{n} d \lambda-\int f_{n}^{2} d \mu & =\sum_{i \leqq a_{n}}\left(i / 2^{n}\right)\left[\left(\lambda-\left(i / 2^{n}\right) \mu\right)\left(L_{i}\right)\right] \\
& >n a_{n}\left(-\varepsilon_{n}\right) \rightarrow 0(\text { see }(7)) .
\end{aligned}
$$

Proof of (v).

$$
\begin{aligned}
\int(H-K)^{2} d \mu= & \int(H-F)^{2} d \mu+\int(F-K)^{2} d \mu \\
& +2\left(\int(H-F)(F-K) d \mu\right) ;
\end{aligned}
$$

and

$$
\begin{aligned}
\int(H-F)(F-K) d \mu= & \int H F d \mu-\int F^{2} d \mu \\
& -\int H K d \mu+\int F K d \mu \geqq 0 .
\end{aligned}
$$

Moreover, (24) also shows that if $K$ can play the role of $F$ in the theorem, then $\int(F-K)^{2}=0$ and, hence, the conditions of the theorem characterize the Radon-Nikodym derivative of $H$. An excellent interpretation of these results can be found in the papers of H. D. Brunk. 
5. A martingale convergence theorem. In this section, we shall establish which features of a martingale convergence theorem carry over to the setting of additive set functions defined on lattices of sets.

Suppose that $\left\{\mathscr{M}_{n}\right\}$ is an increasing sequence of lattices of subsets of $\Omega$, and $\mathscr{C}=\bigcup_{n} \mathscr{L}_{n}$. Then the algebras, $\mathscr{A}_{n}$, of subsets of $\Omega$ that are generated by these lattices increase to the algebra, $\mathscr{A}$, generated by $\mathscr{A l}$.

Suppose that $\lambda$ and $\mu$ are nonnegative, additive set functions defined on $\mathscr{A}$. Denote by $\lambda_{n}$ and $\mu_{n}$ the restrictions of $\lambda$ and $\mu$ to $\mathscr{A}_{n}$. Denote by $F=\left\{f_{n}\right\}$ the Radon-Nikodym derivative of $\lambda$ with respect to $\mu$, and denote by $G_{k}=\left\{g_{k, n}\right\}_{n=1}^{\infty}$ the Radon-Nikodym derivative of $\lambda_{k}$ with respect to $\mu_{k}$.

Because the sequences $\left\{g_{k, n}\right\}_{n}$ are Cauchy sequences in $L_{1}\left(\Omega, \mathscr{A}_{k}, \mu_{k}\right)$ they are also Cauchy sequences in $L_{1}(\Omega, \mathscr{A}, \mu)$

$$
\text { (i.e., } \left.\int_{\Omega}\left|g_{k, m}-g_{k, n}\right| d \mu=\int_{\Omega}\left|g_{k, m}-g_{k, n}\right| d \mu_{k}\right) \text {. }
$$

Hence the equation

$$
H_{k}(E)=\lim _{n} \int_{E} g_{k, n} d \mu,
$$

$E \in \mathscr{A}$

defines an additive extension of $G_{k}$ to $\mathscr{A}$. Notice that $\left\{H_{k}\right\}$ is determined by $\left\{\mathscr{C}_{k}\right\}, \lambda$, and $\mu$.

Now we have enough notation to state our martingale convergence theorem succinctly.

THEOREM. Suppose that $\lambda$ is absolutely continuous with respect to $\mu$. Then the sequence $\left\{H_{k}\right\}$ converges to $F$ in $V_{1}(\Omega, \mathscr{A}, \mu)$.

Before establishing this theorem, let us give a simple example to illustrate the fact that the requirement that $\lambda$ be absolutely continuous with respect to $\mu$ is not superfluous.

ExAmple. Suppose that $\Omega$ is the set of positive integers, and $\mathscr{A}_{n}$ is the algebra of subsets of $\Omega$ comprised of the subsets of the first $n$ positive integers and their complements in $\Omega, n \geqq 1$. Let $\lambda$ be the additive function on $\mathscr{A}$ which assigns zero to a finite set and one to the complement of a finite set, and let $\mu$ be defined on the elements $E$ of $\mathscr{A}$ by $\mu(E)=\sum_{x \in E} 2^{-x}$. Then $\lambda$ is singular with respect to $\mu$; but, for each positive integer $n, \lambda_{n}$ is absolutely continuous with respect to $\mu_{n}$. Suppose that $\mathscr{A}_{n}=\mathscr{A}_{n}, n \geqq 1$. Then

$$
G_{n}=\int 2^{n} \chi_{[n+1, n+2, \ldots]} d \mu,
$$


and the sequence $\left\{H_{n}\right\}$ is not Cauchy.

The example shows that it is possible to have an increasing sequence $\left\{\mathscr{A}_{n}\right)$ of algebras such that $\lambda$ is not absolutely continuous with respect to $\mu$ even though all the $\lambda_{n}$ 's are absolutely continuous with respect to the corresponding $\mu_{n}$ 's. However, $\lambda$ and $\mu$ are determined by the sequences $\left\{\lambda_{n}\right\}$ and $\left\{\mu_{n}\right\}$. Moreover, given sequences $\left\{\lambda_{n}\right\}$ and $\left\{\mu_{n}\right\}$ such that each $\lambda_{n}$ is absolutely continuous with respect to $\mu_{n}$, then $\lambda$ is absolutely continuous with respect to $\mu$ if, and only if, the $\lambda_{n}$ 's are uniformly absolutely continuous with respect to the $\mu_{n}$ 's (i.e., for each $\varepsilon>0$ there is $\delta>0$ such that if $E \in \mathscr{A}_{n}$ and $\mu_{n}(E)<\delta$, then $\left.\lambda_{n}(E)<\varepsilon\right)$.

Proceeding to a proof of the theorem, suppose there is a positive number, $\varepsilon$, and a subsequence $\left\{H_{k_{n}}\right\}$ satisfying $\left\|H_{k_{n}}-F\right\| \geqq 3 \varepsilon, n=$ $1,2, \cdots$ Relabeling if necessary, we can suppose that $k_{n}=n$. Since $F=\left\{f_{i}\right\}$ and the $f_{i}^{\prime}$ 's are simple $\mathscr{A}$-measurable functions, there exists $n_{i}$ such that $f_{i}$ is $\mathscr{A}_{n_{i}}$-measurable. The sequence $\left\{\mathscr{A}_{n}\right\}$ is increasing, so we can take $n_{i+1}>n_{i}$ and look at the corresponding sequence $\left\{H_{n_{i}}\right\}_{i}$. Relabeling again, we can thus suppose that $f_{i}$ is $\mathscr{A}_{i}$-measurable and $\left\|H_{i}-F\right\| \geqq 3 \varepsilon$. Because of the manner in which we defined the sequences $\left\{f_{i}\right\}$ and $\left\{g_{j, i}\right\}_{i}$, we can assume that $g_{j, i}=f_{i}$ for $i \leqq j$. Referring back to the construction, we have $(\lambda-n \mu)\left(M^{c} \cap N\right)>-\varepsilon_{n}$ which implies $\mu\left(M^{c} \cap N\right)<\left(\lambda(\Omega)+\varepsilon_{n}\right) / n$. Hence, from the convergence of $\mu\left(M^{c} \cap N\right)$ to zero, follows the convergence of $\lambda\left(M^{c} \cap N\right)$ to zero. Since the $\lambda_{k}$ 's are uniformly absolutely continuous with respect to the $\mu_{k}^{\prime}$ 's, the corresponding values $\lambda_{k}\left(M_{(k)}^{c} \cap N_{(k)}\right)$ converge to zero uniformly in $k$. (The definitions of $M_{(k)}$ and $N_{(k)}$ are gleaned by putting $\mathscr{C}_{k}$ into the construction.) Checking the paragraph that produced (14) and then checking (15)-(17) permits us to claim that

$$
\lim _{n} G_{k, n}(\Omega)=\lambda_{k}(\Omega)=\lambda(\Omega)
$$

uniformly in $k$. Thus, from (21) and the remark that follows (21) we can conclude that $\lim _{n} G_{k, n}=G_{k}$ uniformly in $k$. Since

$$
F_{k}=\int f_{k} d \mu=\int g_{k k} d \mu, \lim _{k} F_{k}=F,
$$

and

$$
\lim _{k}\left(\left\|\int g_{k, k} d \mu-H_{k}\right\|\right)=0,
$$

we have $\lim _{k}\left(\left\|H_{k}-F\right\|\right)=0$.

CoRollary 1. Suppose that $\mathscr{M}_{n}$ is an increasing sequence of lattices of subsets of $\Omega$. For each positive integer $n$, suppose that 
each of $\lambda_{n}$ and $\mu_{n}$ is a nonnegative additive function defined on $\mathscr{A}_{n}$ such that $\lambda_{n+1} \mid \mathscr{A}_{n}=\lambda_{n}$ and $\mu_{n+1} \mid \mathscr{A}_{n}=\mu_{n}$. Finally, suppose that the $\lambda_{k}$ 's are uniformly absolutely continuous with respect to the $\mu_{k}^{\prime}$ 's. Then the sequence $\left\{H_{k}\right\}$ converges in norm.

We have restricted our attention to nonnegative functions in this paper because we wished to keep the setting simple enough to make our presentation reasonably easy to follow.

The following corollary for an increasing sequence $\left\{\mathscr{A}_{n}\right\}$ of algebras of subsets of $\Omega$ will be established by using the construction given in [2].

CoRollary 2. Suppose that $\left\{\mathscr{A}_{n}\right\}$ is an increasing sequence of algebras of subsets of $\Omega, \lambda$ and $\mu$ are bounded, additive set functions defined on $\mathscr{A}=\mathrm{U}_{n} \mathscr{A}_{n}$, with $\mu$ nonnegative and $\lambda$ is absolutely continuous with respect to $\mu$. For each positive integer $n$, take $\mathscr{C}_{n}=$ $\mathscr{A}_{n}$. Then $\left\{H_{k}\right\}$ converges to $\lambda$ in norm.

Proof. Refer to the construction given in [2]. Adopt the notation of the martingale convergence theorem and repeat the relabelings described in the proof of the martingale convergence theorem. Recall that $F=\lambda$ and $G_{k}=\lambda_{k}, k \geqq 1$, because $\mathscr{M}_{k}=\mathscr{A}_{k}$. Hence

$$
\varepsilon \leqq\left\|H_{k}-F\right\| \leqq\left\|H_{k}-\int g_{k, n} d \mu\right\|+\left\|\int\left(g_{k, n}-f_{k}\right) \mu\right\|+\left\|\int f_{k} d \mu-\lambda\right\| .
$$

But, the first and third terms of the right side of this latter inequality can be made smaller than $\varepsilon$, and the following observations show that the second term can also be made smaller than $\varepsilon$.

$$
\begin{aligned}
\left\|\int\left(g_{k, n}-f_{k}\right) d \mu\right\| & =\int\left|g_{k, n}-f_{k}\right| d \mu=\int\left|g_{k, n}-f_{k}\right| d \mu_{k} \\
& =\left\|\int\left(g_{k, n}-f_{k}\right) d \mu_{k}\right\| \\
& \leqq\left\|G_{k, n}-G_{k}\right\|+\left\|G_{k}-\lambda_{k}\right\|+\left\|\lambda_{k}-f_{k} d \mu_{k}\right\| \\
& \leqq\left\|G_{k, n}-G_{k} \mid+0+\right\| \lambda-\int f_{k} d \mu \| .
\end{aligned}
$$

The version of Corollary 1 that is appropriate for algebras will not be transcribed.

\section{REFERENCES}

1. H. D. Brunk, Conditional expectation given a $\sigma$-lattice and applications, Ann. Math. Statist. 36 (1965), 1339-1350.

2. R. B. Darst, $A$ decomposition of finitely additive set functions, J. Math. Reine Angew. 210 (1962), 31-37. 
3. N. Dunford and J. T. Schwartz, Linear Operators, Vol. 1, Interscience, New York, 1958.

4. S. Johansen, The descriptive approach to the derivative of a set function with respect to a $\sigma$-lattice, Pacific J, Math. 21 (1967), 49-58.

5. S. Leader, The theory of $L^{p}$-spaces for finitely additive set functions, Ann. of Math. 58 (1953), 528-543.

6. B. J. Pettis, On the extension of measures, Ann. of Math. 54 (1981), 186-197.

7. P. Porcelli, Adjoint spaces of abstract $L_{p}$ spaces, Port. Math. 25 (1966), 105-122.

Received July 24, 1969, and in revised form April 10, 1970. This research was supported in part by the National Science Foundation under Grant No. GP-9470.

PURdue University 


\section{PACIFIC JOURNAL OF MATHEMATICS}

\section{EDITORS}

\author{
H. SAMELSON \\ Stanford University \\ Stanford, California 94305 \\ Richard Pierce \\ University of Washington \\ Seattle, Washington 98105
}

\author{
J. DugundJI \\ Department of Mathematics \\ University of Southern California \\ Los Angeles, California 90007 \\ RICHARD ARENS \\ University of California \\ Los Angeles, California 9.0024
}

\section{ASSOCIATE EDITORS}

\begin{tabular}{|c|c|}
\hline E. F. BECKENBACH & K. YoshidA \\
\hline \multicolumn{2}{|c|}{ SUPPORTING INSTITUTIONS } \\
\hline UNIVERSITY OF BRITISH COLUMBIA & STANFORD UNIVERSITY \\
\hline CALIFORNIA INSTITUTE OF TECHNOLOGY & UNIVERSITY OF TOKYO \\
\hline UNIVERSITY OF CALIFORNIA & UNIVERSITY OF UTAH \\
\hline MONTANA STATE UNIVERSITY & WASHINGTON STATE UNIVERSITY \\
\hline UNIVERSITY OF NEVADA & UNIVERSITY OF WASHINGTON \\
\hline NEW MEXICO STATE UNIVERSITY & $* \quad * \quad *$ \\
\hline OREGON STATE UNIVERSITY & AMERICAN MATHEMATICAL SOCIETY \\
\hline UNIVERSITY OF OREGON & CHEVRON RESEARCH CORPORATION \\
\hline OSAKA UNIVERSITY & TRW SYSTEMS \\
\hline UNIVERSITY OF SOUTHERN CALIFORNIA & NAVAL WEAPONS CENTER \\
\hline
\end{tabular}

The Supporting Institutions listed above contribute to the cost of publication of this Journal, but they are not owners or publishers and have no responsibility for its content or policies.

Mathematical papers intended for publication in the Pacific Journal of Mathematics should be in typed form or offset-reproduced, (not dittoed), double spaced with large margins. Underline Greek letters in red, German in green, and script in blue. The first paragraph or two must be capable of being used separately as a synopsis of the entire paper. The editorial "we" must not be used in the synopsis, and items of the bibliography should not be cited there unless absolutely necessary, in which case they must be identified by author and Journal, rather than by item number. Manuscripts, in duplicate if possible, may be sent to any one of the four editors. Please classify according to the scheme of Math. Rev. Index to Vol. 39. All other communications to the editors should be addressed to the managing editor, Richard Arens, University of California, Los Angeles, California, 90024.

50 reprints are provided free for each article; additional copies may be obtained at cost in multiples of 50 .

The Pacific Journal of Mathematics is published monthly. Effective with Volume 16 the price per volume (3 numbers) is $\$ 8.00$; single issues, $\$ 3.00$. Special price for current issues to individual faculty members of supporting institutions and to individual members of the American Mathematical Society: $\$ 4.00$ per volume; single issues $\$ 1.50$. Back numbers are available.

Subscriptions, orders for back numbers, and changes of address should be sent to Pacific Journal of Mathematics, 103 Highland Boulevard, Berkeley, California, 94708.

PUBLISHED BY PACIFIC JOURNAL OF MATHEMATICS, A NON-PROFIT CORPORATION

Printed at Kokusai Bunken Insatsusha (Internatıonal Academic Printing Co., Ltd.), 7-17, Fujimi 2-chome, Chiyoda-ku, Tokyo, Japan. 


\section{Pacific Journal of Mathematics}

\section{Vol. 35, No. $3 \quad$ November, 1970}

John D. Arrison and Michael Rich, On nearly commutative degree one algebras . . . 533

Bruce Alan Barnes, Algebras with minimal left ideals which are Hilbert spaces . . . . 537

Robert F. Brown, An elementary proof of the uniqueness of the fixed point index . . . 549

Ronn L. Carpenter, Principal ideals in F-algebras .................... 559

Chen Chung Chang and Yiannis (John) Nicolas Moschovakis, The Suslin-Kleene

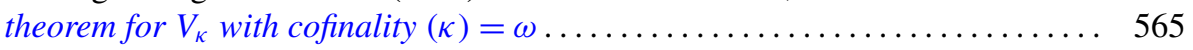

Theodore Seio Chihara, The derived set of the spectrum of a distribution

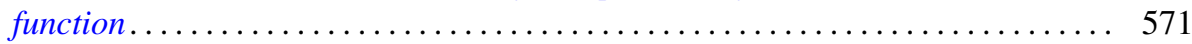

Tae Geun Cho, On the Choquet boundary for a nonclosed subspace of $C(S) \ldots \ldots \quad 575$

Richard Brian Darst, The Lebesgue decomposition, Radon-Nikodym derivative,

conditional expectation, and martingale convergence for lattices of sets .......

David E. Fields, Dimension theory in power series rings . . . . . . . . . . . .

Michael Lawrence Fredman, Congruence formulas obtained by counting

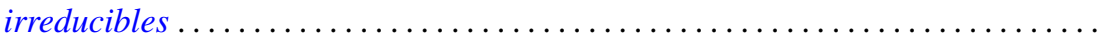

John Eric Gilbert, On the ideal structure of some algebras of analytic functions.....

G. Goss and Giovanni Viglino, Some topological properties weaker than

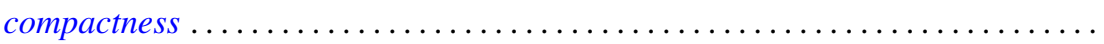

581

601

625

George Grätzer and J. Sichler, On the endomorphism semigroup (and category) of

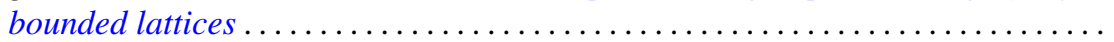

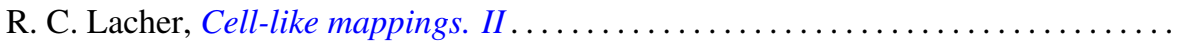

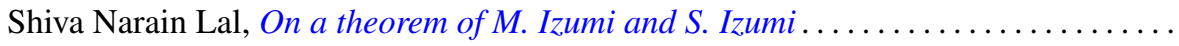

661

Howard Barrow Lambert, Differential mappings on a vector space ...............

Richard G. Levin and Takayuki Tamura, Notes on commutative power joined

semigroups.

Robert Edward Lewand and Kevin Mor McCrimmon, Macdonald's theorem for quadratic Jordan algebras.

J. A. Marti, On some types of completeness in topological vector spaces ....

Walter J. Meyer, Characterization of the Steiner point

717

Saad H. Mohamed, Rings whose homomorphic images are $q$-rings ...

727

Thomas V. O'Brien and William Lawrence Reddy, Each compact orientable surface

of positive genus admits an expansive homeomorphism ...

737

Robert James Plemmons and M. T. West, On the semigroup of binary relations...

743

Calvin R. Putnam, Unbounded inverses of hyponormal operator . .

755

William T. Reid, Some remarks on special disconjugacy criteria for differential

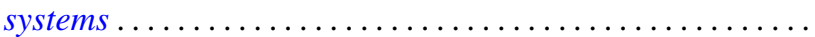

C. Ambrose Rogers, The convex generation of convex Borel sets in euclidean

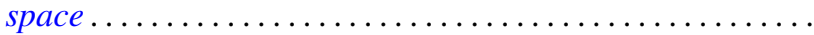

S. Saran, A general theorem for bilinear generating functions .

S. W. Smith, Cone relationships of biorthogonal systems ......

Wolmer Vasconcelos, On commutative endomorphism rings ....

795

Vernon Emil Zander, Products of finitely additive set functions from Orlicz

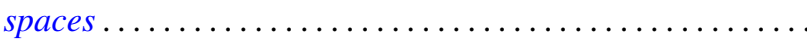

G. Sankaranarayanan and C. Suyambulingom, Correction to: "Some renewal

theorems concerning a sequence of correlated random variables" .

Joseph Zaks, Correction to: "Trivially extending decompositions of $E^{n}$ ”....... 805

Dong Hoon Lee, Correction to: "The adjoint group of Lie groups" ............ 805

James Edward Ward, Correction to: "Two-groups and Jordan algebras". 